\title{
A threshold dose of heavy ion radiation that decreases the oxidative enzyme activity of spinal motoneurons in rats.
}

\section{$\operatorname{AUTHOR}(S)$ :}

Ishihara, Akihiko; Nagatomo, Fumiko; Fujino, Hidemi; Kondo, Hiroyo; Nojima, Kumie

\section{CITATION:}

Ishihara, Akihiko ... [et al]. A threshold dose of heavy ion radiation that decreases the oxidative enzyme activity of spinal motoneurons in rats.. Neurochemical research 2012, 37(2): 387-393

\section{ISSUE DATE:}

2012-02

URL:

http://hdl.handle.net/2433/153976

\section{RIGHT:}

The final publication is available at www.springerlink.com; この論文は 出版社版でありません。引用の際には出版社版をご確認ご利用くださ $\iota_{\circ}$; This is not the published version. Please cite only the published version. 
Neurochemical Research (Original Paper)

\title{
A threshold dose of heavy ion radiation that decreases the oxidative enzyme activity of spinal motoneurons in rats
}

\author{
Akihiko Ishihara $\cdot$ Fumiko Nagatomo $\cdot$ Hidemi Fujino \\ Hiroyo Kondo $\cdot$ Kumie Nojima
}

\author{
A. Ishihara (Correspondence) · F. Nagatomo \\ Laboratory of Neurochemistry, Graduate School of Human and Environmental Studies, Kyoto \\ University, Kyoto 606-8501, Japan \\ e-mail: ishihara@life.mbox.media.kyoto-u.ac.jp \\ H. Fujino
}

Department of Rehabilitation Science, Kobe University Graduate School of Health Sciences, Kobe 654-0142, Japan

H. Kondo

Department of Food Sciences and Nutrition, Nagoya Women's University, Nagoya 467-8610, Japan

K. Nojima

Molecular Imaging Center, National Institute of Radiological Sciences, Chiba 263-8555, Japan

Correspondence: Prof. Akihiko Ishihara

Laboratory of Neurochemistry

Graduate School of Human and Environmental Studies

Kyoto University

Sakyo-ku, Kyoto 606-8501, Japan

E-mail address: ishihara@life.mbox.media.kyoto-u.ac.jp

Tel.: +81 75753 6881; Fax: +81 757536771 


\begin{abstract}
The effect of heavy ion radiation exposure of the spinal cord on the properties of the motoneurons innervating the slow soleus and fast plantaris muscles was investigated. A 15-, 20-, 40-, 50-, or 70-Gy dose of carbon ions (5 Gy/min) was applied to the 2 nd to the 6th lumbar segments of the spinal cord in rats. After a 1-month recovery period, the number and cell body size of the irradiated motoneurons innervating the soleus and plantaris muscles did not differ from that of the non-irradiated controls, irrespective of the dose received. However, the oxidative enzyme activity of these motoneurons was decreased by heavy ion radiation at doses of 40, 50, and 70 Gy compared to that of the non-irradiated controls. This decrease in oxidative enzyme activity levels in the motoneurons returned to that of the non-irradiated controls after a 6-month recovery period. We conclude that heavy ion radiation at doses of 40-70 Gy reversibly decreases the oxidative enzyme activity of motoneurons in the spinal cord of rats.
\end{abstract}

Keywords Heavy ion radiation $\cdot$ Oxidative enzyme activity $\cdot$ Plantaris muscle $\cdot$ Rat $\cdot$ Recovery $\cdot$ Soleus muscle $\cdot$ Spinal motoneuron 


\section{Introduction}

The alpha and gamma motoneurons that innervate the skeletal muscles are located in the dorsolateral region of the ventral horn of the spinal cord [1-3]. Alpha motoneurons are relatively large and innervate the extrafusal fibers of skeletal muscles, whereas gamma motoneurons are relatively small and innervate the intrafusal fibers of muscle spindles $[4,5]$. Alpha motoneurons are classified into slow- and fast-types; slow-type alpha motoneurons have smaller cell body sizes and higher oxidative enzyme activity than fast-type alpha motoneurons $[6,7]$. Slow- and fast-type alpha motoneurons presumably innervate high-oxidative slow-twitch (ST) and low-oxidative fast-twitch (FT) fibers in the skeletal muscles, respectively [8].

Our previous studies [9-12] revealed decreased oxidative enzyme activity in slow-type alpha motoneurons in the spinal cord of rats following a 14-day exposure to microgravity. We also observed decreased oxidative enzyme activity in sensory neurons (relatively large cells of groups Ia and II) in the dorsal root ganglion of rats following the 14-day exposure to microgravity [13]. In addition, after exposure to microgravity, ST fiber atrophy and a fiber type shift from ST to FT were observed in the skeletal muscles, especially the antigravity slow muscles [14]. Therefore, we concluded that slow-type alpha motoneurons and innervating ST fibers associated with antigravity functions are selectively influenced by exposure to microgravity.

Heavy ions are associated with high linear energy transfer (LET); high-LET radiation has been shown to have a greater affect on cells and tissues than low-LET radiation. The risks associated with the exposure of cells and tissues to heavy ions and microgravity have been generally established. However, no data are available on the effects of heavy ion radiation on motoneuron properties. It remains unknown whether there is a critical threshold of heavy ion radiation that is detrimental to motoneurons, which depends on the radiation dose and whether the biological effects of heavy ion radiation on alpha and gamma motoneurons and slow- and fast-type alpha motoneurons differ. In addition, no data are available on the recovery-associated changes in motoneuron properties following heavy ion radiation. We hypothesized that heavy ion radiation at higher doses would cause a greater reduction in the oxidative enzyme activity of motoneurons. Furthermore, we hypothesized that effects on the oxidative enzyme activity induced by heavy ion radiation would differ between different motoneuron types, e.g., alpha and gamma motoneurons or slow- and fast-type alpha motoneurons. 
In the present study, the spinal cords of rats were irradiated with different doses of heavy ions, and the number, cell body size, and oxidative enzyme activity of the motoneurons that innervate the slow soleus and fast plantaris muscles were examined at 1 and 6 months after heavy ion radiation. We used charged particle (carbon) beams, which have greater biological effects on target cells, but cause less damage to neighboring tissues.

\section{Experimental Procedure}

All experimental procedures were conducted in accordance with the National Institute of Health Guide for the Care and Use of Laboratory Animals. This study was approved by the Institutional Animal Care and Use Committee of Kyoto University (Kyoto, Japan).

Experimental Animals and Heavy Ion Radiation

Eight-week-old male Wistar rats were subjected to a single dose of radiation with a carbon ion beam $(290 \mathrm{MeV}, 5 \mathrm{~mm}$ SOBP, LET $=130 \mathrm{keV} / \mu \mathrm{m})$ using the Heavy Ion Medical Accelerator in Chiba (HIMAC) at the National Institute of Radiological Sciences (NIRS), Japan. A 15-, 20-, $40-, 50-$, or 70-Gy dose of carbon ions ( $5 \mathrm{~Gy} / \mathrm{min}$ ) was applied to the 2 nd to 6 th lumbar segments of the spinal cord without surgical treatment ( $n=5$ for each dose level). Five age-matched rats served as controls. All rats were housed for 1 month after heavy ion radiation.

A 70-Gy dose of carbon ions was also applied to the spinal cord of a second group $(n=5)$ as described above; however, in this experiment, the rats were housed for 6 months after heavy ion radiation. Five age-matched rats served as controls.

All rats were maintained in a controlled environment with a 12:12 h light:dark cycle (light on from 0800 to 2000 ), and temperature and relative humidity were maintained at $22 \pm 2{ }^{\circ} \mathrm{C}$ and $45-55 \%$, respectively. Food and water were provided ad libitum for all rats.

\section{Injection of Fluorescent Neuronal Tracer}

The rats were anesthetized with sodium pentobarbital ( $5 \mathrm{mg} / 100 \mathrm{~g}$ body weight), and the surgical procedures were performed under aseptic conditions. A $2 \%$ fluorescent neuronal tracer, nuclear yellow, which has been shown to be retrogradely transported from the skeletal muscle to the spinal cord, was injected using a microsyringe into multiple sites of the left soleus and right plantaris muscles $[8,15]$. Care was taken to inject the neuronal tracer slowly, so as to prevent leakage. After injecting the neuronal tracer, the skin was sutured and the rats were allowed to recover from the anesthesia and surgery. The rats were sacrificed by administering an overdose of sodium pentobarbital 2 days after injection of the neuronal tracer. 
Histochemical Analyses

The lumbosacral enlargement of the spinal cord was removed and frozen immediately in isopentane cooled in liquid nitrogen. Serial longitudinal sections $(10-\mu \mathrm{m}$ thick) of the lumbosacral enlargement were cut in a cryostat at $-20^{\circ} \mathrm{C}$. The motoneurons innervating the soleus and plantaris muscles were identified by the golden-yellow fluorescence of the nucleus imparted by nuclear yellow (Fig. 1a). The motoneurons in the serial sections were identified and counted. These same sections that used to identify the motoneurons were subsequently stained for succinate dehydrogenase (SDH) activity, a marker of mitochondrial oxidative capacity (Fig. 1b) $[16,17]$. SDH activity was determined by incubating the sections in a medium containing $100 \mathrm{mM}$ phosphate buffer, $0.9 \mathrm{mM}$ 1-methoxyphenazine methylsulfate, 1.5 $\mathrm{mM}$ nitroblue tetrazolium, $5.6 \mathrm{mM}$ ethylenediaminetetraacetic acid disodium salt, and $48 \mathrm{mM}$ succinate disodium salt $(\mathrm{pH}$ 7.6) for $10 \mathrm{~min}$. The reaction was stopped by multiple washes in distilled water. Sections were then dehydrated in a graded series of ethanol and passed through xylene before a cover slip was applied. The cross-sectional area and SDH activity of the identified motoneurons, in which the nucleus was visible, were measured using a computer-assisted image processing system (Neuroimaging System Inc., Kyoto, Japan). Sectional images were digitized as gray-scale images. Each pixel was quantified as 1 of 256 gray levels; a gray level of 0 was equivalent to $100 \%$ light transmission, whereas a gray level of 255 was equivalent to $0 \%$ light transmission. The mean optical density (OD) of all pixels (which were converted to gray-level values) within a motoneuron was determined using a calibration photographic tablet with 21 steps of gradient-density ranges and the corresponding diffused density values. The nucleus was excluded from OD measurements since it does not stain for SDH activity.

The cut-off for the distinction between alpha and gamma motoneurons was based on cell body size. There is a high probability that the smaller motoneurons $\left(<451 \mu \mathrm{m}^{2}\right)$ are gamma and the larger motoneurons are alpha $[5,7]$.

\section{Statistics}

The mean and standard deviation were calculated from individual values using standard procedures. One-way analysis of variance (ANOVA) was used to evaluate significant differences among groups. When the differences were significant based on ANOVA analyses, further comparisons were made using Scheffé's post hoc tests. A probability $(P)$ value of 0.05 was considered significant. 

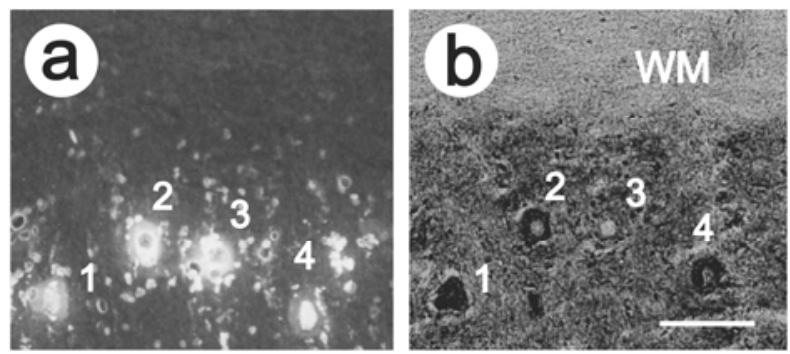

Fig. 1 Spinal motoneurons innervating the rat plantaris muscle were retrogradely labeled by the fluorescent neuronal tracer, nuclear yellow (a). After identification of the motoneurons using nuclear yellow, these 4 motoneurons were stained for succinate dehydrogenase activity (b). $\mathrm{WM}$, white matter. Scale bar $=100 \mu \mathrm{m}$

\section{Results}

The average number and cell body size of motoneurons innervating the soleus and plantaris muscles after 1 month of recovery from heavy ion radiation did not differ from that of the non-irradiated controls, irrespective of the dose received (Table 1). Similarly, after a 1-month recovery period, the average oxidative enzyme activity of motoneurons innervating the soleus (Fig. 2) and plantaris (Figs. 3 and 4) muscles irradiated with 15 and 20 Gy did not differ from that of the non-irradiated controls. In contrast, after a 1-month recovery period, the oxidative enzyme activity of the motoneurons innervating these muscles that were irradiated with 40, 50, and 70 Gy decreased compared to that of the non-irradiated controls.
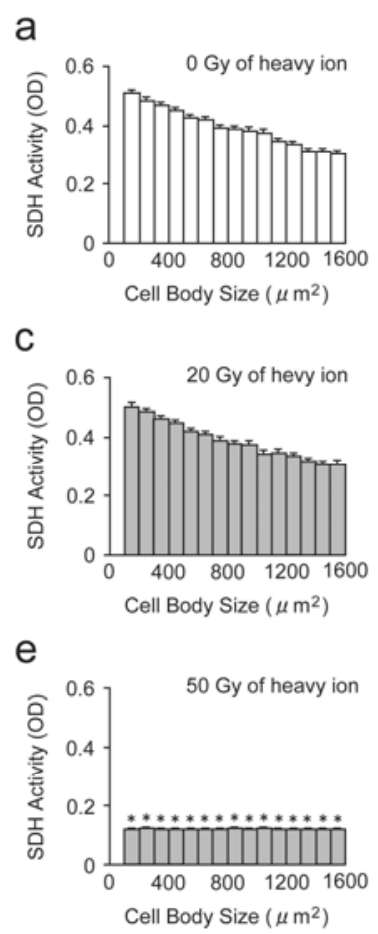

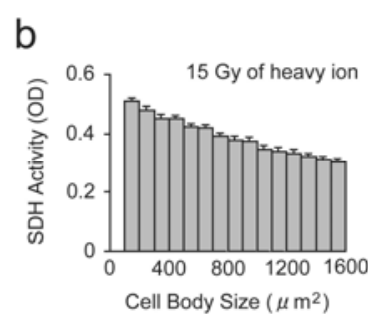

\section{d}
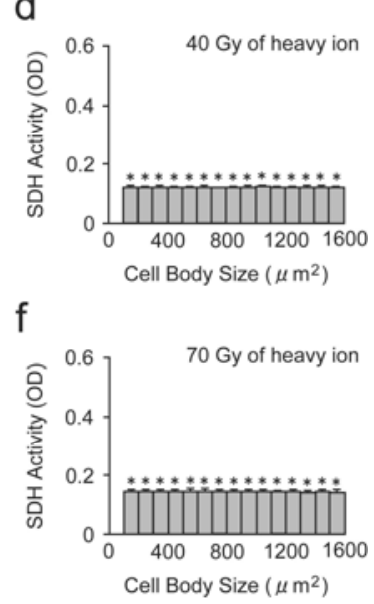

Fig. 2 Succinate dehydrogenase activity in the motoneurons of the spinal cord innervating the soleus muscle in rats after 1 month of recovery from a single dose of radiation with a carbon ion beam at 0 (a), 15 (b), 20 (c), 40 (d), 50 (e), or 70 (f) Gy. The values shown are expressed as the mean and standard deviation $(n=5)$. SDH, succinate dehydrogenase; OD, optical density. ${ }^{*} P$ $<0.05$ compared to the values at the corresponding cell body sizes in $\mathrm{a}, \mathrm{b}$, and $\mathrm{c}$ 

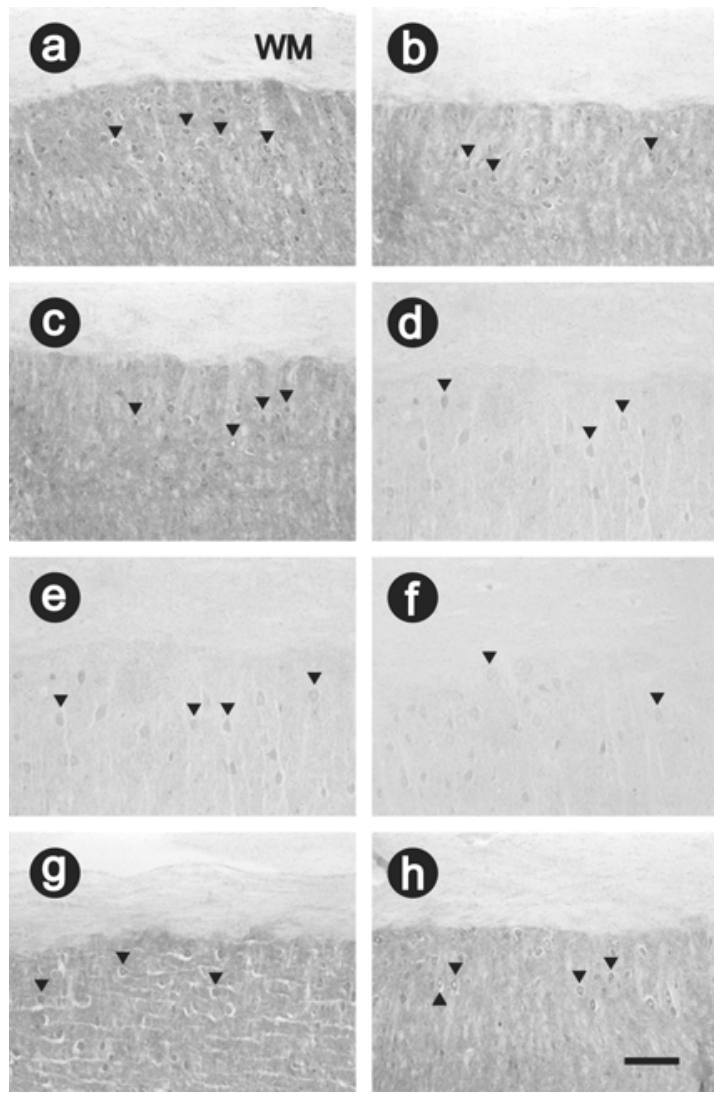

a
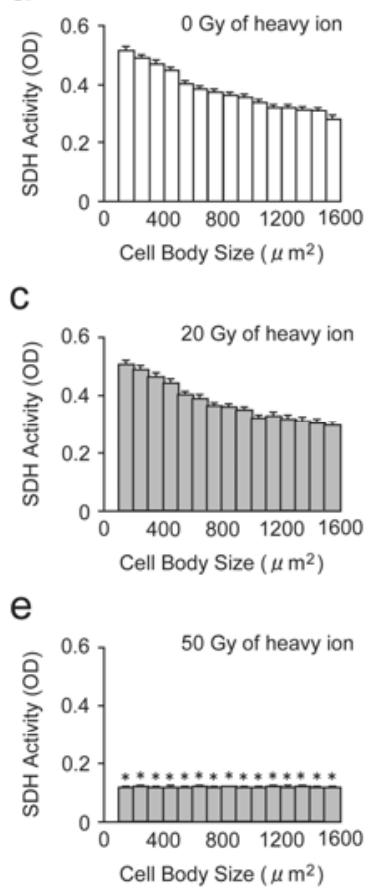
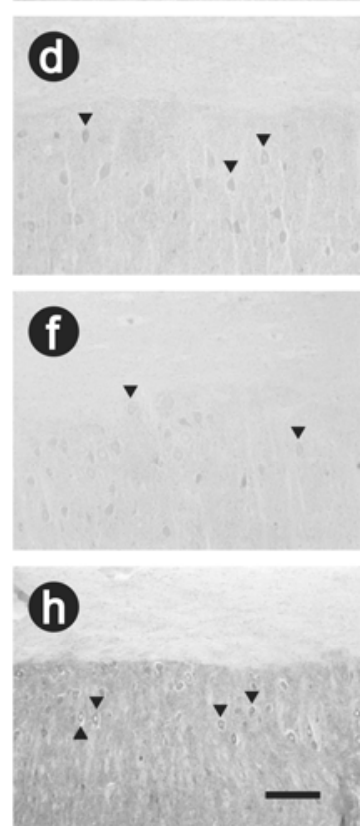

$\boldsymbol{\nabla}$

b

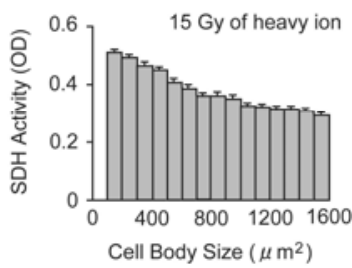

d

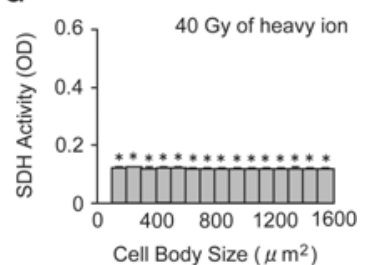

f

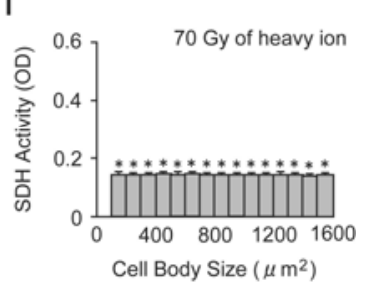

Fig. 3 Spinal motoneurons (arrowheads) innervating the plantaris muscles of rats after 1 month of recovery from a single dose of radiation with a carbon ion beam of 0 (a), 15 (b), 20 (c), 40 (d), 50 (e), or 70 (f) Gy, and after 6 months of recovery from a single dose of radiation with a carbon ion beam of 0 (g) or 70 (h) Gy. WM, white matter. Scale bar $=200 \mu \mathrm{m}$

Fig. 4 Succinate dehydrogenase activity in the motoneurons of the spinal cord innervating the plantaris muscle in rats after 1 month of recovery from a single dose of radiation with a carbon ion beam at 0 (a), 15 (b), 20 (c), 40 (d), 50 (e), or 70 (f) Gy. The values shown are expressed as the mean and standard deviation $(n=5)$. SDH, succinate dehydrogenase; OD, optical density. ${ }^{*} P$ $<0.05$ compared to the values at the corresponding cell body sizes in $\mathrm{a}, \mathrm{b}$, and c

At 6 months after a 70-Gy dose of heavy ion radiation, the average number and cell body size of motoneurons innervating the soleus and plantaris muscles did not differ from that of the non-irradiated controls (Table 1). However, after a 6-month recovery period, the decreased 
oxidative enzyme activity of the motoneurons innervating these muscles that were irradiated with 70 Gy was restored to levels similar to that of the non-irradiated controls, (Figs. 3 and 5).

a
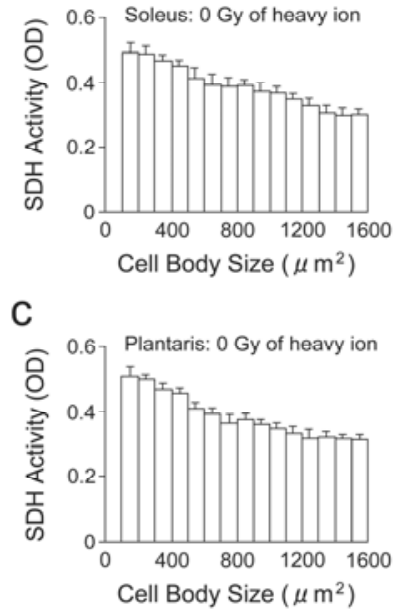

b

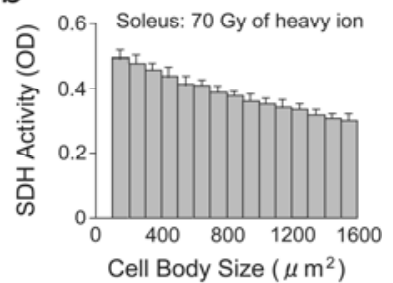

d

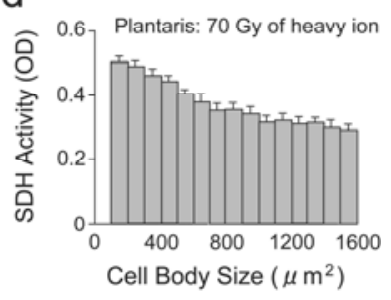

Fig. 5 Succinate dehydrogenase activity in the motoneurons of the spinal cord innervating the soleus $(\mathrm{a}, \mathrm{b})$ and plantaris $(\mathrm{c}$, d) muscles in rats after 6 months of recovery from a single dose of radiation with a carbon ion beam at $0(a, c)$ or $70(b, d)$ Gy. The values shown are expressed as the mean and standard deviation $(n=5)$. SDH, succinate dehydrogenase; OD, optical density

\section{Discussion}

Motoneurons innervating skeletal muscle fibers are distributed throughout the dorsolateral region of the ventral horn in the spinal cord, and exhibit wide variations in cell body size and oxidative enzyme activity [6-8]. There is an inverse relationship between cell body size and oxidative enzyme activity in motoneurons innervating a particular skeletal muscle; smaller motoneurons innervating the soleus, extensor digitorum longus, and tibialis anterior muscles in rats have higher oxidative enzyme activity than larger motoneurons [16, 18-21]. The higher oxidative enzyme activity in smaller motoneurons corresponds well with higher densities of mitochondria [22]. In an alpha motoneuron population, slow-type motoneurons, presumably innervating high-oxidative fibers, have smaller cell body sizes and higher oxidative enzyme activity than fast-type motoneurons, presumably innervating low-oxidative fibers [6-8]. The higher oxidative enzyme activity in smaller motoneurons is appropriate for these more easily recruited motoneurons; smaller motoneurons are recruited before and more often than larger motoneurons and are more metabolically active. The higher oxidative enzyme activity in the smaller motoneurons in rats 1 month after heavy ion radiation at doses of 0 (Figs. 2a and 4a), 15 (Figs. 2b and 4b), and 20 (Figs. 2c and 4c) Gy and 6 months after heavy ion radiation at doses of 0 (Fig. 5a, c) and 70 (Fig. 5b, d) Gy was consistent with our previous findings [16, 18-21].

The heavy ion beam used in the present study is a type of radiation that prevents damage to neighboring tissues, and thus enabled us to examine the biological effects of heavy ion radiation on a narrow and restricted region. Gamma ray and fast neutron beams are strongest 
near the surface of the body and are attenuated in the deep areas of the tissues. Therefore, when a deep region is treated with this type of radiation, cells and tissues between the surface and the target area are susceptible to damage. Deep regions beyond the target are also affected, which presents a difficulty when attempting to clarify the biological effects in a specific region without the mutual interaction with other regions. In contrast, the use of protons or charged particle beams reduces the damage to other cells and tissues by focusing the peak of the beam on the target location; the beam becomes markedly intense at a certain depth according to the energy applied, but is weak at shallower and deeper depths.

The purpose of the present study was to evaluate the heavy ion radiation-induced damage to motoneurons in the spinal cord by measuring the number, cell body size, and oxidative enzyme activity of the motoneurons innervating specific skeletal muscles, i.e., the slow soleus and fast plantaris muscles. We hypothesized that higher doses of heavy ion radiation would induce a greater reduction in the oxidative enzyme activity of motoneurons. However, we found a threshold at which biological effects occur; heavy ion radiation at doses higher than 40 Gy induced a marked decrease in the oxidative enzyme activity of motoneurons innervating the soleus (Fig. 2) and plantaris (Fig. 4) muscles. It is interesting to note that decreased oxidative enzyme activity was observed in all motoneurons, including the alpha and gamma motoneurons and the slow- and fast-type alpha motoneurons. Our previous studies [9-12, 23-26] showed that the oxidative enzyme activity of specific types of motoneurons is decreased or increased in a variety of conditions, such as exposure to hypobaric hypoxia, hyperbaric oxygen, and microgravity. Exposure to hypobaric hypoxia caused an increase in the oxidative enzyme activity of slow-type alpha motoneurons innervating high-oxidative fibers in the slow soleus and fast extensor digitorum longus muscles of rats [23, 24]. In contrast, exposure to microgravity caused a decrease in the oxidative enzyme activity of slow-type alpha motoneurons innervating high-oxidative fibers in the skeletal muscles of rats [9-12]. Exposure to hyperbaric oxygen caused an increase in the oxidative enzyme activity of slow- and fast-type alpha, but not gamma, motoneurons that innervate the slow soleus and fast plantaris muscles of rats $[25,26]$. These results suggest that certain types of motoneurons are specifically susceptible to heavy ion radiation. However, in the present study, heavy ion radiation at doses of 40-70 Gy reduced the oxidative enzyme activity of all motoneurons (Figs. 2 and 4). Heavy ion radiation at doses higher than $40 \mathrm{~Gy}$ affected the oxidative enzyme activity of all motoneurons, including alpha and gamma motoneurons and both slow- and fast-type alpha motoneurons, suggesting that the change induced by heavy ion radiation is not due to adaptation of motoneurons. 
It has been suggested that the decrease in the oxidative enzyme activity of motoneurons can be restored if the changes are not due to apoptosis, and if the time period between irradiation and sacrifice is sufficient to allow recovery. Therefore, we examined the effects of 70 Gy of heavy ion radiation (the maximum dose used in the present study) on motoneuron properties after a 6-month recovery period. The decreased oxidative enzyme activity of the motoneurons innervating the soleus and plantaris muscles induced by heavy ion radiation was restored to normal levels after 6 months of recovery from heavy ion radiation (Fig. 5). This result indicates that the changes in motoneuron oxidative enzyme activity induced by heavy ion radiation are reversible.

In conclusion, we found a critical threshold for the heavy ion radiation dose that induces detrimental effects in the motoneurons of the spinal cord in rats; heavy ion radiation at doses higher than 40 Gy induces a reduction in the oxidative enzyme activity of motoneurons. Furthermore, we found that these changes in the oxidative enzyme activity of motoneurons induced by heavy ion radiation are reversible; the decreased oxidative enzyme activity of motoneurons was restored to normal levels after 6 months of recovery from heavy ion radiation.

Acknowledgments This study was supported by grants for use of the Heavy Ion Medical Accelerator in Chiba (HIMAC) awarded by the National Institute of Radiological Sciences (NIRS), Japan (No.14B244). We are grateful to Dr. Shunji Nagaoka (Department of Physiology, Fujita Health University, Toyoake, Japan) for critical advice and discussions. The authors declare that there is no duality of interest associated with this manuscript.

\section{References}

1. McHanwell S, Biscoe TJ (1981) The localization of motoneurons supplying the hindlimb muscles of the mouse. Philos Trans R Soc Lond B Biol Sci 293:477-508

2. Nicolopoulos-Stournaras S, Iles JF (1983) Motor neuron columns in the lumbar spinal cord of the rat. J Comp Neurol 217:75-85

3. Peyronnard JM, Charron LF, Lavoie J et al (1986) Motor, sympathetic and sensory innervations of rat skeletal muscles. Brain Res 373:288-302

4. Johnson IP (1986) A quantitative ultrastructural comparison of alpha and gamma motoneurons in the thoracic region of the spinal cord of the adult cat. J Anat 147:55-72 
5. Swett JE, Wikholm RP, Blanks RHI et al (1986) Motoneurons of the rat sciatic nerve. Exp Neurol 93:227-252

6. Burke RE, Dum RP, Fleshman JW et al (1982) An HRP study of the relation between cell size and motor unit type in cat ankle extensor motoneurons. J Comp Neurol 209:17-28

7. Sickles DW, Oblak TG (1984) Metabolic variation among $\alpha$-motoneurons innervating different muscle-fiber types. I. Oxidative enzyme activity. J Neurophysiol 51:529-537

8. Ishihara A, Roy RR, Edgerton VR (1995) Succinate dehydrogenase activity and soma size of motoneurons innervating different portions of the rat tibialis anterior. Neuroscience $68: 813-822$

9. Ishihara A, Ohira Y, Roy RR et al (1996) Influence of spaceflight on succinate dehydrogenate activity and soma size of rat ventral horn neurons. Acta Anat 157:303-308

10. Ishihara A, Ohira Y, Roy RR et al (2000) Comparison of the response of motoneurons innervating peripheral and hind limb muscles to spaceflight and recovery. Muscle Nerve $23: 753-762$

11. Ishihara A, Ohira Y, Roy RR et al (2002) Succinate dehydrogenase activity in rat dorsolateral ventral horn motoneurons at L6 after spaceflight and recovery. J Grav Physiol 9:39-48

12. Ishihara A, Yamashiro J, Matsumoto A et al (2006) Comparison of cell body size and oxidative enzyme activity in motoneurons between the cervical and lumbar segments in the rat spinal cord after spaceflight and recovery. Neurochem Res 31:411-415

13. Ishihara A, Ohira Y, Roy RR et al (1997) Effects of 14 days of spaceflight and nine days of recovery on cell body size and succinate dehydrogenase activity of rat dorsal root ganglion neurons. Neuroscience 81:275-279

14. Roy RR, Baldwin KM, Edgerton VR (1996) Response of neuromuscular unit to spaceflight: what has been learned from the rat model? Exerc Sports Sci Rev 24:399-425

15. Ishihara A, Taguchi S, Araki H et al (1991) Retrograde neuronal labeling of motoneurons in the rat by fluorescent tracers, and quantitative analysis of oxidative enzyme activity in labeled neurons. Neurosci Lett 124:141-143

16. Nagatomo F, Ishihara A, Ohira Y (2009) Effects of hindlimb unloading at early postnatal growth on cell body size in spinal motoneurons innervating soleus muscle of rats. Int J Dev Neurosci 27:21-26

17. Nagatomo F, Ishihara A, Sudoh M et al (2011) Growth-related changes in motoneuron properties of rats are not influenced by chronic exposure to 2-G. J Grav Physiol, in press. 
18. Ishihara A, Ohira Y, Tanaka M et al (2001) Cell body size and succinate dehydrogenase activity of spinal motoneurons innervating the soleus muscle in mice, rats, and cats. Neurochem Res 26:1301-1304

19. Ishihara A, Kawano F, Ishioka N et al (2003) Growth-related changes in cell body size and succinate dehydrogenase activity of spinal motoneurons innervating the rat soleus muscle. Int J Dev Neurosci 21:461-469

20. Ishihara A, Kawano F, Ishioka $\mathrm{N}$ et al (2004) Effects of running exercise during recovery from hindlimb unloading on soleus muscle fibers and their spinal motoneurons in rats. Neurosci Res 48:119-127

21. Huang F, Shimizu T, Ishihara A et al (2005) Tissue hyperoxygenation promotes oxidative metabolism in motor unit. J Neurosci Res 80:584-591

22. Ishihara A, Hayashi S, Roy RR et al (1997) Mitochondrial density of ventral horn neurons in the rat spinal cord. Acta Anat 160:248-253

23. Ishihara A, Taguchi S, Itoh M et al (1990) Oxidative metabolism of the rat soleus neuron pool following hypobaric hypoxia. Brain Res Bull 24:143-146

24. Taguchi S, Ishihara A, Itoh M et al (1990) Effects of hypobaric hypoxia on the oxidative capacity of the extensor digitorum longus motor units in the rat. Neurochem Res $15: 923-926$

25. Ishihara A, Kawano F, Okiura T et al (2005) Hyperbaric exposure with high oxygen concentration enhances oxidative capacity of neuromuscular units. Neurosci Res $52: 146-152$

26. Matsumoto A, Okiura T, Morimatsu F et al (2007) Effects of hyperbaric exposure with high oxygen concentration on the physical activity of developing rats. Dev Neurosci 29:452-459 
Table 1 Number and cell body size of motoneurons innervating the soleus and plantaris muscles in rats

Soleus motoneurons

\begin{tabular}{|c|c|c|c|c|c|}
\hline \multicolumn{4}{|c|}{ Number } & \multicolumn{2}{|c|}{ Cell body size $\left(\mu \mathrm{m}^{2}\right)$} \\
\hline & Gamma & Alpha & $\%$ Alpha & Gamma & Alpha \\
\hline \multicolumn{6}{|c|}{ After 1 month of recovery } \\
\hline Control & $18 \pm 4$ & $42 \pm 5$ & $70.1 \pm 5.7$ & $326 \pm 12$ & $890 \pm 16$ \\
\hline 15 Gy & $17 \pm 4$ & $40 \pm 6$ & $69.4 \pm 6.0$ & $325 \pm 13$ & $886 \pm 17$ \\
\hline 20 Gy & $15 \pm 3$ & $41 \pm 6$ & $73.5 \pm 5.8$ & $327 \pm 13$ & $895 \pm 19$ \\
\hline 40 Gy & $18 \pm 4$ & $39 \pm 5$ & $68.2 \pm 5.4$ & $321 \pm 14$ & $897 \pm 18$ \\
\hline 50 Gy & $17 \pm 4$ & $38 \pm 5$ & $68.5 \pm 5.2$ & $325 \pm 14$ & $882 \pm 19$ \\
\hline 70 Gy & $19 \pm 4$ & $43 \pm 5$ & $69.4 \pm 5.1$ & $328 \pm 15$ & $896 \pm 18$ \\
\hline \multicolumn{6}{|c|}{ After 6 months of recovery } \\
\hline Control & $17 \pm 4$ & $44 \pm 5$ & $72.4 \pm 6.0$ & $330 \pm 20$ & $894 \pm 17$ \\
\hline $70 \mathrm{~Gy}$ & $19 \pm 4$ & $43 \pm 4$ & $69.9 \pm 6.3$ & $327 \pm 15$ & $899 \pm 19$ \\
\hline
\end{tabular}

Plantaris motoneurons

\begin{tabular}{|c|c|c|c|c|c|}
\hline & \multicolumn{3}{|l|}{ Number } & \multicolumn{2}{|c|}{ Cell body size $\left(\mu \mathrm{m}^{2}\right)$} \\
\hline & Gamma & Alpha & $\%$ Alpha & Gamma & Alpha \\
\hline \multicolumn{6}{|c|}{ After 1 month of recovery } \\
\hline Control & $18 \pm 4$ & $67 \pm 6$ & $79.0 \pm 4.7$ & $319 \pm 15$ & $908 \pm 20$ \\
\hline 15 Gy & $19 \pm 4$ & $66 \pm 5$ & $77.8 \pm 4.5$ & $316 \pm 12$ & $900 \pm 23$ \\
\hline 20 Gy & $17 \pm 4$ & $69 \pm 5$ & $80.3 \pm 3.6$ & $319 \pm 13$ & $909 \pm 22$ \\
\hline 40 Gy & $20 \pm 4$ & $65 \pm 5$ & $77.0 \pm 2.7$ & $318 \pm 14$ & $916 \pm 22$ \\
\hline 50 Gy & $16 \pm 4$ & $66 \pm 5$ & $80.4 \pm 3.9$ & $315 \pm 15$ & $899 \pm 21$ \\
\hline 70 Gy & $19 \pm 4$ & $68 \pm 5$ & $77.9 \pm 2.6$ & $311 \pm 14$ & $907 \pm 24$ \\
\hline \multicolumn{6}{|c|}{ After 6 months of recovery } \\
\hline Control & $18 \pm 4$ & $71 \pm 6$ & $79.7 \pm 3.4$ & $317 \pm 12$ & $911 \pm 20$ \\
\hline 70 Gy & $16 \pm 4$ & $70 \pm 7$ & $81.6 \pm 3.6$ & $314 \pm 14$ & $908 \pm 23$ \\
\hline
\end{tabular}

mean $\pm \operatorname{SD}(n=5)$. 